\title{
SIMULTANEOUS UBVRIJK PHOTOMETRIC AND POLARIMETRIC OBSERVATIONS OF PQ GEM
}

\author{
STEPHEN POTTER ${ }^{1}$, K. O. MASON ${ }^{1}$, M. S. CROPPER ${ }^{1}$, \\ J. A. BAILEY ${ }^{2}$, J. H. HOUGH ${ }^{3}$ \\ 1. Mullard Space Science Laboratory, Holmbury St. Mary, \\ Dorking, Surrey, RH5 6NT, England \\ 2. Anglo-Australian Observatory, PO Box 296, Epping, \\ NSW 2121, Australia \\ 3. Division of Physical Sciences, University of Hertfordshire, \\ Herts, AL10 9AB, England
}

We present simultaneous multiwavelength observations of the intermediate polar PQ Gem (Mason et al. 1992) obtained on 1993 February 18 and 19 using the Hatfield Polarimeter on UKIRT.

The data are folded on the $13.9 \mathrm{~m}$ spin period in Fig. 1. The light curves are double peaked at long wavelengths, with dips at phase $\sim 0.15$ and phase $\sim 0.65$, but almost sinusoidal in the $U$ and $B$ bands where the phase $\sim 0.65$ dip is absent. The percentage of circular polarisation also varies with the spin cycle, most notably in the $I$ band, with both positive and negative excursions. The peaks in the positive and negative polarisation occur at phase $\sim 0.15$ and phase $\sim 0.65$ respectively, approximately coincident with the two intensity dips.

We have modelled these light curves using arc-shaped emission regions. The spectrum of each point along the arc is calculated using the data of Meggitt \& Wickramasinghe (1982) and the spectrum summed as a function of spin phase for various viewing geometries. We have used $\Lambda \sim 10^{5}$ (where $\Lambda$ is the optical depth parameter), $k T \sim 10 \mathrm{keV}$ models with a field of $\sim 18$ $\mathrm{MG}$ at the magnetic pole. The best fit to the observations was obtained for a system inclination of $60^{\circ}$ and magnetic dipole offset of $30^{\circ}$, with two extended arcs located at opposite ends of the magnetic dipole (Fig. 2).

Our model explains the dip in the negative polarisation at phase $\sim 0.65$ as absorption or scattering of the cyclotron radiation by the accretion column as the accretion region traverses the line of sight. Half a cycle later the main emission region is occulted by the white dwarf resulting in the wavelength independent dip at phase $\sim 0.15$. 


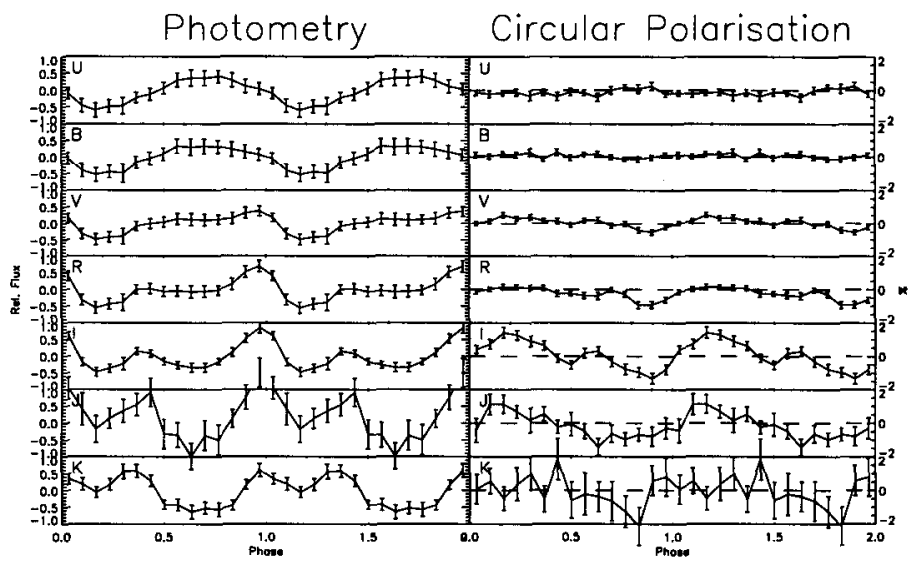

Figure 1. Photometry and circular polarimetry of PQ Gem plotted against the phase of the $13.9 \mathrm{~m}$ spin period with the ephemeris $2449297.973+0.009645966 \mathrm{~N}$.

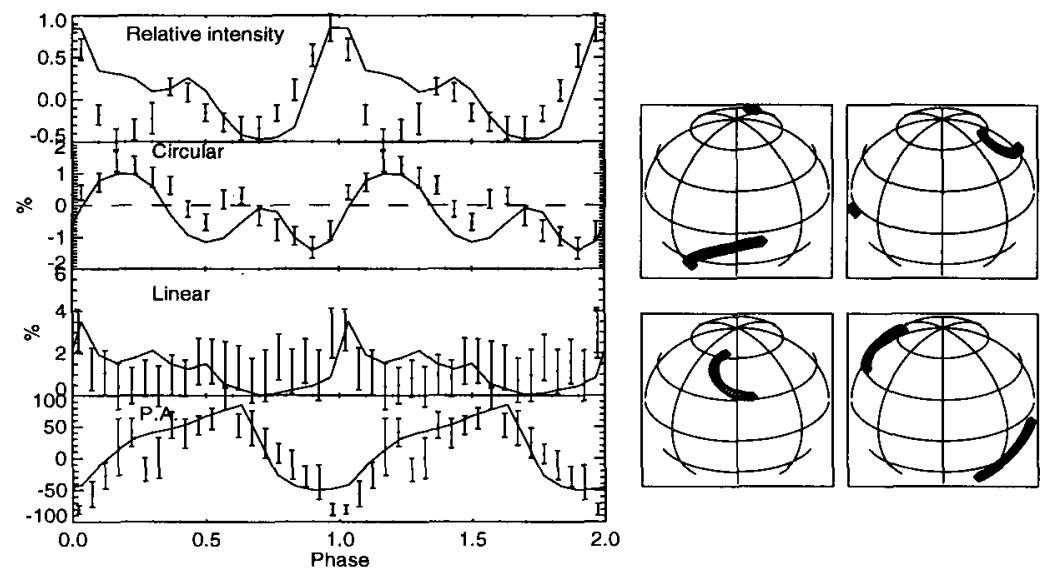

Figure 2. Model fit to the $I$ band photometry and circular polarisation. The linear and position angle observations taken from Pïrola, Hakala \& Coyne (1993). Best fit obtained for an inclination of $60^{\circ}$ and with the magnetic dipole offset at an angle of $30^{\circ}$.

\section{References}

Mason, K.O., Watson, M.G., Ponman T.J., et al., 1992, MNRAS, 258, 794

Meggitt, S.M., Wickramasinghe, D.T., 1982, MNRAS, 198, 71

Piirola, V., Hakala, P., Coyne, G.V., 1993, Ap. J., 410, L107 